Revue internationale P.M.E.

Économie et gestion de la petite et moyenne entreprise

\title{
Des réseaux de l'entrepreneur aux ressorts du créatif : quelles stratégies pour les territoires?
}

\section{Raphaël Suire}

Volume 17, numéro 2, 2004

URI : https://id.erudit.org/iderudit/1008460ar

DOI : https://doi.org/10.7202/1008460ar

Aller au sommaire du numéro

Éditeur(s)

Presses de l’Université du Québec

ISSN

0776-5436 (imprimé)

1918-9699 (numérique)

Découvrir la revue

Citer cette note

Suire, R. (2004). Des réseaux de l'entrepreneur aux ressorts du créatif : quelles stratégies pour les territoires? Revue internationale P.M.E., 17(2), 123-143. https://doi.org/10.7202/1008460ar
Résumé de l'article

Cet article propose un cadre d'analyse original du comportement entrepreneurial dès lors que celui-ci repose sur un double encastrement : encastrement social d'abord, encastrement territorial ensuite. Nous posons les fondements de l'innovation disruptive comme reposant sur le triptyque confiance - capital social - créativité. L'articulation des liens sociaux forts et des liens sociaux faibles est au cœur de ce processus. À mesure que les liens faibles apparaissent prégnants, les territoires doivent s'engager dans des stratégies de mise à disposition de ressources relationnelles, d'une part, soit des institutions locales favorisant la confiance et, d'autre part, soutenir et promouvoir les conditions d'absorption de ces ressources par la mise à disposition de lieux physiques où échanger.
Tous droits réservés @ Presses de l’Université du Québec, 2004
Ce document est protégé par la loi sur le droit d'auteur. L'utilisation des services d'Érudit (y compris la reproduction) est assujettie à sa politique d'utilisation que vous pouvez consulter en ligne.

https://apropos.erudit.org/fr/usagers/politique-dutilisation/ 


\title{
Des réseaux de l'entrepreneur aux ressorts du créatif: quelles stratégies pour les territoires?
}

Raphaël SUIRE*

Université de Rennes I

MOTS CLÉS
Capital social - Réseau social - Liens forts-faibles
Interactions stochastiques - Innovation disruptive
Entrepreneuriat - Attractivité territoriale

\begin{abstract}
RÉSUMÉ
Cet article propose un cadre d'analyse original du comportement entrepreneurial dès lors que celui-ci repose sur un double encastrement: encastrement social d'abord, encastrement territorial ensuite. Nous posons les fondements de l'innovation disruptive comme reposant sur le triptyque confiance - capital social -créativité.
\end{abstract}

\section{L'AUTEUR}

RAPHAËL SUIRE détient un doctorat en économie de l'Université de Rennes I ; il est actuellement chercheur associé au Centre de recherche rennais en économie et en gestion (UMR CNRS 6585) et membre du Môle armoricain de recherche sur la société de l'information et les usages de l'Internet (MARSOUIN). Ses travaux de recherche portent fondamentalement sur l'économie des interactions et sur la dynamique des réseaux sociaux. Ils sont appliqués à l'analyse de l'impact des TIC sur la géographie des activités et la dynamique des clusters d'activités TIC, aux fondements des comportements innovants et, enfin, à l'analyse des comportements des consommateurs sur l'Internet. Adresse: Faculté des sciences économiques, Université de Rennes I, 7, place Hoche, F35065 Rennes, Cédex, France. Courriel : <raphael.suire @univ-rennes1.fr>.

* L'auteur remercie les rapporteurs anonymes de la revue pour leurs commentaires et reste, bien entendu, seul responsable des erreurs, omissions ou imprécisions qui pourraient subsister. 
L'articulation des liens sociaux forts et des liens sociaux faibles est au cœur de ce processus. À mesure que les liens faibles apparaissent prégnants, les territoires doivent s'engager dans des stratégies de mise à disposition de ressources relationnelles, d'une part, soit des institutions locales favorisant la confiance et, d'autre part, soutenir et promouvoir les conditions d'absorption de ces ressources par la mise à disposition de lieux physiques où échanger.

\begin{abstract}
This paper proposes an original analytical framework of entrepreneurship behaviour. This one relies on a double embedment: a social embedment and a territorial embedment. We propose that disruptive innovation is a crucial function of trust social capital - creativity triptych. The mix of strong ties and weak ties is at the heart of this process. As a matter of fact, weak ties are pregnant, therefore, territories must have a double objective of development. The first one deals with relational assets and local institutions that favour inter-relational trust, the second one is about the physical places that allowed exchange of ideas and individual absorption of these resources.
\end{abstract}

\title{
RESUMEN
}

Este articulo propone un marco de anàlisis original del comportamiento del espíritu emprededor en cuanto éste descansa sobre un doble encaje : encaje social primero, encaje territorial después. Los fundamentos de la innovación disruptiva se establecen en el triptico confianza - capital social - creatividad. La articulación de los vinculos sociales fuertes y de los vinculos sociales débiles està en el corazon de este proceso. A medida que los vinculos débiles se imponen, los territorios han de meterse en las estrategias de puesta a disposición de recursos relacionales por una parte, o sea instituciones locales que favorecen la confianza y por otra parte soportar y promover las condiciones de absorciones de estos recursos con la puesta a disposición de lugares físicos en los que se puede intercambiar.

\section{ZUSAMMENFASSUNG}

Dieser Artikel bietet einen Analyserahmen für das unternehmerische Verhalten, basierend auf dem sozialen und räumlichen Gefüge der Unternehmung. Die Grundlagen der disruptiven, nicht nachhaltigen, Innovation beruhen unseres Erachtens auf Vertrauen, sozialen Kompetenzen und Kreativität. Die Verknüpfung von starken sozialen mit schwachen sozialen Verbindungen ist der Kern des beschriebenen Vorganges. Sollten die sozialen Verbindungen nur schwach bestehen, müssen sich die öffentlichen Institutionen einerseits um Strategien bemühen, die die Zurverfügungsstellung von Ressourcen aus dem Beziehungsnetz ermöglichen. Andererseits sind Orte und Räumlichkeiten notwendig, damit die Ressourcen aufgenommen und genutzt werden können. 


\section{Introduction}

Beaucoup ont vu, et à juste titre d'ailleurs, l'innovation technologique comme étant le ressort fondamental d'une économie fondée sur l'accumulation des connaissances (Foray, 2000). Le propos n'est pas ici de s'étendre sur ce qu'est l'innovation, sur ce que sont ses formes, ses modalités de diffusion et/ou d'appropriation ou encore son impact macroéconomique, nous souhaitons plutôt ici aborder et soulever un point relatif au comportement innovant, à savoir son double encastrement. Notamment, nous soutenons l'idée que le comportement disruptif est le produit d'un encastrement socio-technico-économique et d'une inscription territoriale de ces réseaux. Dès lors, les territoires doivent redessiner les contours de leur stratégie dans le sens d'une attractivité et d'une rétention mieux définies.

Depuis les travaux de Granovetter (2000) ou encore ceux de Schelling (1978), nous comprenons mieux ce qu'il advient des comportements lorsque ceux-ci subissent la contrainte ou l'influence de voisinage. Réunis au sein d'une «économie des interactions » (Cohendet et al., 1998; Kirman et Zimmermann, 2001 ; Dalle et Vicente, 2001 ; Suire, 2002), nous savons désormais mieux qualifier les comportements microéconomiques d'adoption et / ou de déclenchement, notamment du point de vue des mécanismes d'interactions sociales, mais aussi les conséquences agrégées et collectives de ces attitudes lorsque les « autres comptent »(David, 1988) pour la décision individuelle.

Nous ne nous interrogerons pas sur l'usage de cette méthodologie (agents hétérogènes en interactions rationalité limitée et située) ainsi que sur ses enjeux épistémologiques (dépassement du cadre strict de l'agent représentatif et reconsidération de l'institution du marché comme mécanisme unique de coordination des comportements économiques [Kirman, 1992]) mais plutôt sur un champ d'application très peu développé à notre connaissance, soit celui des comportements innovants. Plus justement, il s'agit de s'interroger sur ce qui fonde un comportement innovant et d'en dériver des conséquences quant aux stratégies territoriales de développement. Pour le dire en quelques mots, il s'agit de s'interroger sur la boucle formée par les éléments suivants : ... $<->$ réseau social $<->$ confiance $<->$ capital social $<->$ créativité $<->$ performance territoriale $<->$...(voir figure 1$)$.

Chaque relation causale doit ici s'entendre au sens d'une condition nécessaire qui reste insuffisante. En effet, à aucun moment la créativité, pour ne citer qu'elle, ne peut se déduire de la seule existence du capital social ${ }^{1}$, elle reste le plus souvent la résultante d'un processus cognitif complexe, voire d'un mécanisme spontané. En revanche, si le capital social, vu comme une ressource d'abord

1. Entendu comme l'ensemble des ressources informationnelles tirées de l'appartenance à un réseau social (voir, par exemple, Coleman, 1988). 
FIGURE 1

\section{Causalités circulaires et dynamique}

du double encastrement de l'innovation

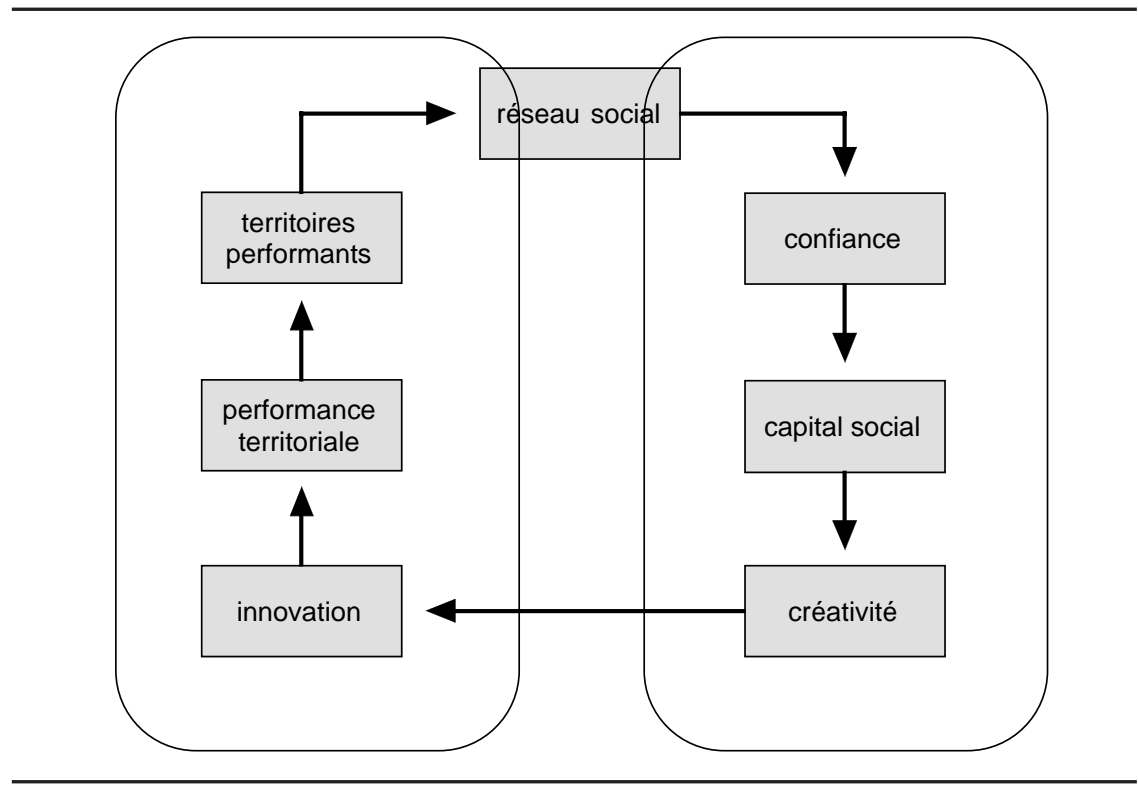

participant de la créativité individuelle puis comme participant à un processus d'innovation disruptif est abordé par la littérature (Baker, 2000), les rapports entretenus plus généralement entre ces comportements individuels innovants et les déterminants spatiaux font l'objet de très peu de réflexions. Si l'objectif de cet article est de jeter un éclairage sur les ressorts fondamentaux de la créativité et de la rupture, il est aussi question de montrer quelle position, non moins fondamentale, doivent tenir les territoires dans ce processus de créativité. De ce point de vue, nous discernons les territoires performants comme étant ceux qui mettent à la disposition des innovateurs des actifs relationnels et des institutions économiques et sociales qui favorisent la mise en confiance tout en limitant au maximum les barrières à l'entrée pour des comportements et des idées en rupture.

Nous reviendrons dans la première section (1) sur une définition. Il va s'agir pour nous de définir le capital social comme une ressource tirée d'une interaction sociale. Puis nous verrons comment ces relations sociales et le capital social structurent les projets innovants (2). Cela nous amènera à présenter plus formellement la dynamique des réseaux (3) et ce qu'est une structure d'interactions sociales au sens de la théorie des «petites mondes» (small worlds) de Watts (1999). Après avoir observé que la capacité à innover dépend du type et de la morphologie des 
relations sociales entretenues (4) et ayant relevé les insuffisances de cette modélisation, nous proposerons les simulations d'un modèle original d'agents hétérogènes en interaction qui permettent d'illustrer l'articulation entre confiance, capital social et créativité (5). Les conjectures que nous posons permettent de décrire les fondements de l'innovation disruptive que nous définissons comme un comportement créatif en rupture. Cela nous permet d'envisager l'action territoriale dans une perspective de valorisation des actifs relationnels, ceux qui favorisent l'échange et la diffusion d'idées ou de routines comportementales hétérogènes (6).

\section{Le capital social : de quoi parle-t-on?}

Nous proposons, pour plus de clarté, de réduire l'ensemble des ressources informationnelles qu'un entrepreneur peut retirer de ses interactions hors marchés, à son capital social. Ainsi, peut-on définir deux sources d'information pour un entrepreneur individuel. L'une est publique et transite par les marchés (cabinets de conseils, expertises privées, revues spécialisées, etc.), l'autre est privé et propre aux systèmes d'interactions singuliers de l'entrepreneur (réseau d'affaires, relations personnelles, clubs, etc.). Le capital social est, par conséquent, une profonde source de différenciation et d'hétérogénéité comportementale. Mais ayant dit cela se pose désormais la question de savoir si l'on doit assimiler ce capital social à un flux informationnel ou plutôt à un ensemble fermé de ressources prenant dans ce cas la forme d'un stock.

Les réponses apportées à cette question changent profondément la nature de ce que l'on cherche et, a fortiori, de ce que l'on trouve. Faut-il tenter d'établir les causes ou les effets attendus du capital ? Selon que l'on privilégie l'une ou l'autre des définitions, les méthodes à mettre en œuvre diffèrent et les conclusions peuvent diverger. Si Woolcock (2001, p. 11) plaide largement pour qu'une définition du capital social «mette l'accent sur ses sources, plutôt que sur ses conséquences, c'est-à-dire sur ce qu'est le capital social plutôt que sur ce qu'il fait », notre propre analyse repose à l'inverse sur l'assimilation du capital social à une variable de stock. En effet, ce qui nous importe, c'est de définir les formes de capital social qui favorisent la créativité et l'innovation disruptive et non pas la manière dont chaque individu investit dans ses relations sociales pour se doter d'un capital social (Glaeser, Laibson et Sacerdote, 2001)². En définitive, nous rejoignons la définition de Coleman (1988) et considérons le capital social comme une ressource individuelle qui contribue, par un effet multiplicateur sur les autres formes de capital individuel, à différencier les individus quant à leur capacité à innover.

2. Dans ce dernier cas, la littérature idoine a plus à voir avec la question de la morphogenèse des réseaux.

Revue internationale P.M.E., vol. 17, $\mathrm{n}^{\mathrm{o}}$ 2, 2004 


\section{De l'invention collective à l'invention collective spatialisée}

L'invention collective (Allen, 1983) illustre la manière dont la dynamique du réseau d'interrelations favorise (ou ne favorise pas) l'apparition d'un surplus collectif de connaissance. Récemment, les travaux fondés sur le cadre formel des «petits mondes » de Watts (1999), Watts et Strogatz (1998), repris par Cowan et Jonard (2000), Cowan, Jonard et Zimmermann (2003) pour formaliser l'idée de Allen, ont permis de se faire une idée assez précise de la relation entre la topologie ou la structure d'interaction (reposant typiquement sur un compromis entre interactions locales et interactions globales) et les modalités de diffusion d'un signal ou d'une information au sein d'une structure sociale.

Pour Allen, l'invention collective se nourrit du principe de circulation et d'échange d'informations, elles-mêmes reposant sur l'existence d'un réseau de cliques sociales (des groupes sociaux cohérents et homogènes). Il faut donc comprendre comment se forment et évoluent les cliques sociales (dynamique intracommunautaire) mais aussi comment ces dernières interagissent entre elles (dynamique intercommunautaire), chacune étant toujours susceptible de rétroagir sur l'autre, pour définir la manière dont émerge une invention collective.

C'est l'étude de l'industrie des hauts fourneaux de l'Angleterre du milieu du XIX ${ }^{\mathrm{e}}$ siècle qui amène Allen à ce type de réflexions. À cette époque, les producteurs partageaient assez facilement leurs connaissances sur le mode de fonctionnement des fourneaux, aussi, et collectivement, cela leur a-t-il permis, chemin faisant, de faire émerger une relation empirique assez juste entre la taille du fourneau et le niveau de production. Il n'est pas le lieu ici de rappeler nombre d'exemples de ce type où le partage de connaissances a favorisé l'émergence d'une dynamique collective vertueuse et compétitive ${ }^{3}$, cependant, on peut s'arrêter un instant sur ce que cela implique d'ores et déjà à mesure que l'espace et la localisation des agents sont introduits dans le raisonnement.

L'émergence de grappes d'activités compétitives comme peut l'être la Silicon Valley (Saxenian, 1994) ou comme le sont (l'étaient?) les districts italiens sont des illustrations manifestes d'inventions collectives spatialisées. Ici se combinent une dynamique locale des agents et une performance globale de la grappe d'activités au sens où le territoire favorise la circulation des idées, la diffusion des innovations et le développement technologique. Ayant dit cela, posons-nous la question de savoir s'il existe des modalités d'interactions qui favorisent au mieux la circulation des idées et la diffusion des innovations. En un mot et pour faire écho à ce

3. Voir Von Hippel (1988) parmi d'autres.

Revue internationale P.M.E., vol. 17, nº 2, 2004 
que nous avons énoncé sur le capital social : existe-t-il une forme singulière de relations interpersonnelles qui favorise l'échange des idées et l'innovation? La récente littérature sur les «petits mondes » nous éclaire utilement à cet égard.

\section{Dynamique des réseaux}

La diffusion de l'information entre les agents est le facteur clé de l'invention collective. La nature des liens qui supportent la transmission, soit l'émission et la réception d'une information, ainsi que l'organisation des liens entre eux sont donc déterminants ${ }^{4}$.

Le grand intérêt du travail de Watts (1999) est d'avoir mis en lumière une structure d'interactions singulière dont les propriétés remarquables en font un mode d'organisation sociétale performant. Partant du principe que l'échange d'information se fait entre deux agents connectés et considérant que ces deux agents sont proches (dans une acception large, c'est-à-dire culturelle, géographique, etc.), on peut définir une structure limite définissant une organisation collective singulière que l'on nomme réseau régulier (figure 2). À l'inverse, lorsque j'interagis plus facilement à distance, c'est-à-dire que je privilégie un collègue éloigné plutôt que mon voisin de bureau, dans ce cas, l'organisation collective forme un réseau aléatoire (figure 2). Ces deux structures ne sont pas neutres quant à la circulation de l'information. En effet, une information circule potentiellement moins rapidement dans le premier cas, alors que sa diffusion s'en trouve accélérée dans le second. Pour voir cela, il suffit d'envisager un choc informationnel, exogène à la structure, à l'égard d'un agent du réseau. Si, dans le premier cas, il faut un temps constant pour que l'ensemble des agents du réseau intègre la nouvelle information, en revanche, pour le réseau aléatoire, le temps de la diffusion à l'ensemble de la structure va être très fortement contingent à la combinaison des liens locaux et des liens globaux des agents qui subissent le choc. En un mot, à la forte cohésion sociale de la première configuration (cliquishness) s'oppose une plus grande accessibilité en tous points du réseau (path length) pour la seconde configuration. L’information se diffuse de proche en proche dans un réseau régulier et sa diffusion est plutôt irradiante dans le second cas.

Il existe un continuum de structures d'interactions entre ces deux configurations, mais l'une se distingue par ses propriétés remarquables, c'est celle des «petits mondes». Elle présente le meilleur compromis entre cohésion locale et accessibilité globale au réseau.

4. Pour une vision stratégique de la constitution des réseaux, voir Jackson et Wolinsky (1996) et Bala et Goyal (2000).

Revue internationale P.M.E., vol. 17, nº 2, 2004 
FIGURE 2

Structures d'interactions de type «petits mondes»
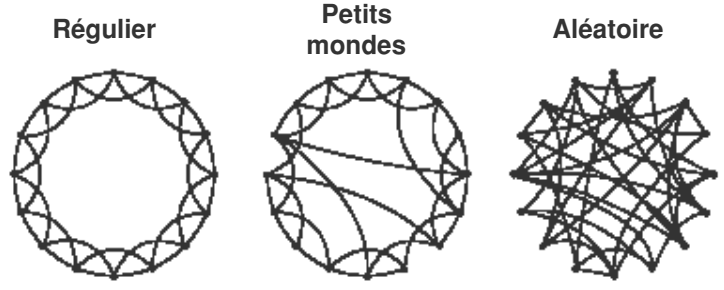

$p=0$

Connections aléatoires

$p=1$

Source: Watts (1999, p. 68).

Cowan et Jonard (2000) montrent que, si cette configuration est celle qui présente la meilleure performance au regard de la vitesse du taux d'accumulation de connaissance, en revanche, elle est aussi source d'inégalité dès lors qu'il est question de distribuer les nouvelles connaissances sur l'ensemble des agents. Collectivement, cette structure apparaît efficiente, mais, individuellement, elle discrimine profondément les agents selon qu'ils ont des interactions locales/globales ou seulement locales.

De ce point de vue, la nature des interactions d'un agent, ou dit autrement, la composition du capital social, apparaît déterminante dès lors qu'il est question d'obtenir «au plus vite» de nouvelles informations. Toutefois, et s'agissant de réfléchir à ce que peut être un comportement en rupture, non seulement le stock individuel d'information, déterminé par une articulation singulière de liens locaux et de liens globaux, compte, mais doit aussi compter la capacité individuelle à gérer ce stock dont dépend, in fine, l'innovation ${ }^{5}$.

\section{Les espaces sociaux de l'innovation}

\subsection{Espace social}

La proximité physique favorise incontestablement la constitution de réseaux, euxmêmes vecteurs de diffusion des externalités informationnelles mais bien évidemment, être proche ne suffit pas pour interagir intentionnellement. Autrement dit et pour reprendre la figure 1, le modèle des «petits mondes » propose seulement de définir une relation entre réseau social, capital social et capacité à innover. Ainsi,

5. Cela signifie notamment qu'avec un capital social identique deux agents peuvent avoir des comportements innovants divergents. 
comme indiqué sur la figure 3, deux dimensions importantes de l'interaction sociale sont «oubliées». D'une part, la confiance: elle est le produit d'une forme d'apprentissage et de la mémoire des interactions passées. Elle est déterminante pour valider l'information plutôt que les informateurs, particulièrement lorsque dans un processus d'innovation la réactivité compte. Mais compte aussi la créativité, préalable à une innovation disruptive, qui seule permet en définitive de gagner durablement. Aussi, pouvons-nous dire que l'espace social des «petits mondes » qui sous-tend la genèse d'un capital social performant est donc réduit à une portion très congrue.

FIGURE 3

La dynamique sociale des «petits mondes»

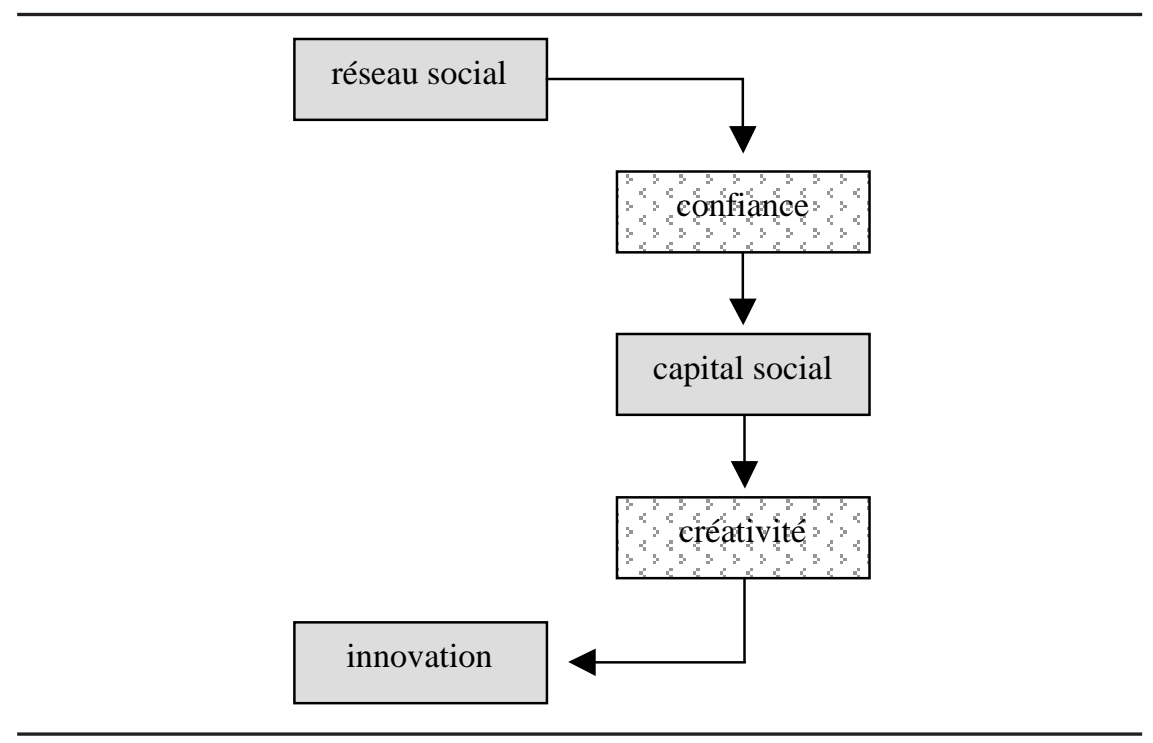

En reprenant deux études récentes sur l'entrepreunariat individuel, il va cependant être possible d'établir les conditions de densification de l'espace social de l'innovation.

\subsection{Composition du capital social et capacité à innover : l'articulation entre liens faibles et liens forts}

Deux études récentes, l'une de Ruef (2002), la seconde de Julien, Andriambeloson et Ramangalahy (2002) ont cherché à tester les hypothèses de Granovetter sur la prégnance des liens faibles sur les liens forts dans la détermination du comportement. Les auteurs cherchent à cerner le mix liens faibles et liens forts qui déclenche l'innovation. 
À partir d'une définition des liens faibles comme ceux que l'on tisse avec l'extérieur d'une communauté d'appartenance ou de pratique et des liens forts comme les interactions typiquement intracommunautaires, il est possible de décliner cette définition dans le cadre des «petits mondes». En un sens, ce sont les liens à distance qui jouent le rôle des liens faibles alors que les liens forts sont définis par les interactions locales. Cela nous permet de reformuler la problématique de Ruef (2002) et de Julien, Andriambeloson et Ramangalahy (2002) comme une recherche de la composition du capital social individuel qui détermine l'innovation ou encore comme une localisation de la structure sous-jacente sur le continuum des structures d'interactions (figure 2).

Le travail de Ruef se fonde sur l'idée shumpétérienne que l'innovation trouve sa source dans une combinaison originale d'idées et de routines existantes. Dès lors, il cherche à rendre compte de l'influence de la structure d'interaction interpersonnelle sur la capacité à innover. Soit, en définitive, sur la capacité à pratiquer des combinaisons originales de routines et d'idées existantes à partir de ses relations sociales.

Nous l'avons dit, l'analyse repose sur la distinction faite entre les liens sociaux faibles et les liens forts. La notion de liens forts renvoie aux liens tissés au sein d'un groupe social homogène et cohérent (une famille, les amis, une ethnie, etc.), ils relèvent de la dynamique intracommunautaire et nécessitent du temps pour s'établir tandis que les liens faibles relèvent de la dynamique intercommunautaire (les amis de mes amis et, plus généralement, les connaissances de mes connaissances, etc.), sont moins gourmands en temps et le plus souvent à l'origine de nouvelles rencontres. Cependant, ces liens faibles sont d'une importance considérable puisqu'ils favorisent la captation d'informations en provenance de l'extérieur d'une clique sociale. En un sens, ils favorisent les comportements de rupture ou du moins préviennent les risques de conformisme. À l'inverse, les agents qui en sont dépourvus s'inscrivent le plus souvent dans des comportements routiniers et des pratiques très faiblement innovantes. Ce type de relations, faibles, est stylisé par les liens à distance dans le réseau des «petits mondes ».

Les liens forts sont moins discriminants et ont plus valeur de bien public, au sens où la ressource informationnelle est distribuée plus rapidement à l'ensemble des agents qui partagent ce communautarisme. En revanche, ils favorisent les effets de conformisme et de polarisation rapide. Autrement dit, tout le monde se heurte au risque «d'avoir la même idée au même moment». Avec ces relations intracommunautaires, tout ce qui semble s'écarter trop radicalement de la routine est alors susceptible de soulever la désapprobation de un ou plusieurs membres du groupe. De ce point de vue, la dimension affective des relations sociales peut souvent être un frein aux comportements atypiques et déviants. Ces relations sociales s'assimilent aux liens locaux du modèle des «petits mondes » et, en définitive, favorisent très peu les combinaisons originales, les expérimentations nouvelles et la créativité. 
Ruef pose l'hypothèse que la capacité à innover est d'autant plus importante que les idées se diffusent à travers les liens faibles. Un modèle probit, testé sur 766 entrepreneurs homogènes ${ }^{6}$, cherche à valider cette hypothèse. Dans $52 \%$ des cas, la mobilisation des liens faibles a été déterminante pour l'innovation, dans $38 \%$ des cas, ce sont les liens forts qui ont joué et, enfin, le discours d'experts a déclenché le comportement innovant dans $19 \%$ des cas. L'étude permet de ne pas rejeter l'hypothèse de la prégnance des liens faibles dans la détermination d'un comportement innovant. Ces relations sociales favorisent l'adaptabilité à des situations nouvelles puisque l'information sur de nouvelles opportunités y est plus facilement accessible.

L'étude de Julien, Andriambeloson et Ramangalahy (2002) concerne le comportement innovant des PME du secteur des équipements de transport terrestre. Ici aussi, l'étude cherche à vérifier comment l'articulation entre liens faibles et liens forts influe sur l'occurrence d'une pratique innovante. Les auteurs cherchent eux aussi à tester l'hypothèse de la supériorité des liens faibles sur les liens forts. Cent quarante-sept firmes sont concernées par l'étude. Sur l'ensemble de cet échantillon, il apparaît clairement que les firmes les plus innovantes sont celles qui recourent plus volontiers aux liens faibles. En d'autres termes, la seule observation du comportement de son voisinage proche ou de ses concurrents est insuffisante pour escompter une innovation disruptive.

Si désormais nous validons que, pour être créatif, il est préférable d'avoir une prise avec l'extérieur, c'est-à-dire un capital social où les liens faibles priment les liens forts, il reste à déterminer quelle est la mécanique sociale qui permet d'augmenter les chances d'être créatif. Autrement dit, comment faire en sorte que le capital social devienne majoritairement composé de liens faibles? Pour cela, nous allons présenter un modèle original qui permet de faire le lien entre créativité, confiance et dynamique intercommunautaire.

\section{Confiance et créativité : un modèle simple et exploratoire}

\subsection{Hypothèses ${ }^{7}$}

Nous considérons une population de $\mathrm{N}$ agents localisés sur un tore fermé ${ }^{8}$ noté $\mathrm{S}$ (figure 4). Chaque agent se trouve en interaction directe avec ses quatre plus proches voisins (figure 5) et retire du réseau social ainsi constitué une ressource que nous nommons « capital social».

6. Typiquement un niveau MBA.

7. Nous reprenons ici les grandes lignes d'un modèle plus général exposé dans Suire (2002).

8. Cette hypothèse permet d'éviter les effets de bords, dès lors que sur un tore ouvert, comme sur un échiquier, les agents situés aux frontières n'ont que trois voisins. 
FigURE 4

Tore fermé $\mathbf{S}$ dans $\mathbf{Z}^{2}$

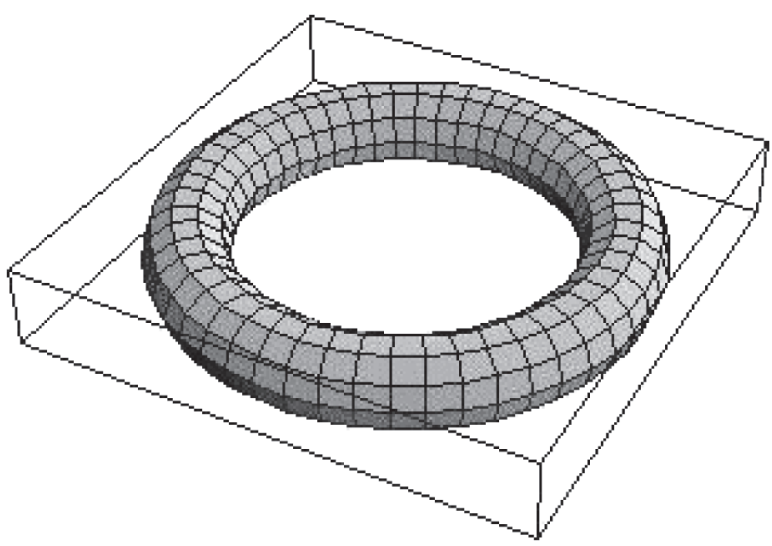

FIGURE 5

Voisinage d'interaction individuel de type Von Neumann

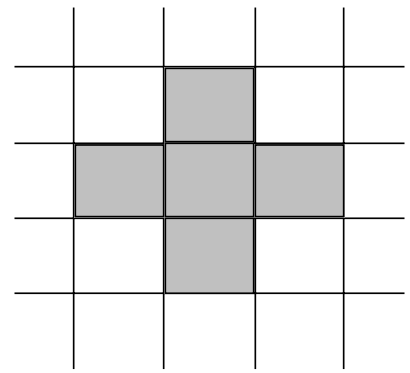

Ce capital social est, comme nous l'avons vu, une ressource nécessaire à l'engagement d'une pratique innovante. Cette dernière, dans sa dimension disruptive, est subordonnée au principe schumpétérien de combinaison originale de routines ${ }^{9}$ existantes. Dans ce modèle simple, nous considérons qu'il existe deux routines parfaitement symétriques : $r_{0}$ et $r_{l}$. Chaque agent est porteur exclusif d'une seule routine et il est dans un état singulier à chaque pas de temps, nous notons :

9. Au sens large, c'est-à-dire une idée, un comportement...

Revue internationale P.M.E., vol. 17, n 2, 2004 


$$
\eta_{\mathrm{i}, t}=\left\{\begin{array}{l}
0 \text { si porteur d'une routine } \mathrm{r}_{0} \\
1 \text { si porteur d'une routine } \mathrm{r}_{1}
\end{array}\right.
$$

soit l'état de l'agent $i$ à l'instant $t$.

De ces hypothèses, nous dénombrons cinq configurations possibles du capital social. Selon la composition du voisinage d'interaction, nous avons en effet:

$$
\begin{aligned}
& \mathrm{C}_{1}=\left(\begin{array}{llll}
\mathrm{r}_{0} & \mathrm{r}_{0} & \mathrm{r}_{0} & \mathrm{r}_{0}
\end{array}\right) \\
& \mathrm{C}_{2}=\left(\mathrm{r}_{1} \mathrm{r}_{1} \mathrm{r}_{1} \mathrm{r}_{1}\right) \\
& \mathrm{C}_{3}=\left(\mathrm{r}_{0} \mathrm{r}_{1} \mathrm{r}_{1} \mathrm{r}_{1}\right) \\
& \mathrm{C}_{4}=\left(\mathrm{r}_{0} \mathrm{r}_{0} \mathrm{r}_{1} \mathrm{r}_{1}\right) \\
& \mathrm{C}_{5}=\left(\begin{array}{llll}
\mathrm{r}_{0} & \mathrm{r}_{0} & \mathrm{r}_{0} & \mathrm{r}_{1}
\end{array}\right)
\end{aligned}
$$

Nous distribuons ensuite ces configurations sur un treillis carré et fermé. La figure 6 représente la composition individuelle du capital social sur un treillis de dimension 10 x 10 . Les porteurs d'une routine $r_{0}$ sont en blanc à droite du treillis alors que les porteurs d'une routine $r_{1}$ sont en noir, à gauche. Les simulations numériques ont été effectuées à partir de treillis de dimension plus importante, 512 x 400 et avec, par conséquent, un nombre $\mathrm{N}=204800$ d'agents (figure 7).

FIGURE 6

Treillis carré et fermé de dimension 10 x 10

\begin{tabular}{|l|l|l|l|l|l|l|l|l|l|}
\hline 1 & 1 & 1 & 1 & 1 & 0 & 0 & 0 & 0 & 0 \\
\hline 1 & 1 & 1 & 1 & 1 & 0 & 0 & 0 & 0 & 0 \\
\hline 1 & 1 & 1 & 1 & 1 & 0 & 0 & 0 & 0 & 0 \\
\hline 1 & 1 & 1 & 1 & 1 & 0 & 0 & 0 & 0 & 0 \\
\hline 1 & 1 & 1 & 1 & 1 & 0 & 0 & 0 & 0 & 0 \\
\hline 1 & 1 & 1 & 1 & 1 & 0 & 0 & 0 & 0 & 0 \\
\hline 1 & 1 & 1 & 1 & 1 & 0 & 0 & 0 & 0 & 0 \\
\hline 1 & 1 & 1 & 1 & 1 & 0 & 0 & 0 & 0 & 0 \\
\hline 1 & 1 & 1 & 1 & 1 & 0 & 0 & 0 & 0 & 0 \\
\hline 1 & 1 & 1 & 1 & 1 & 0 & 0 & 0 & 0 & 0 \\
\hline
\end{tabular}

Revue internationale P.M.E., vol. 17, $\mathrm{n}^{\mathrm{o}}$ 2, 2004 
FIGURE 7

Composition des voisinages et capital social sur un treillis carré et fermé de dimension $512 \times 400$

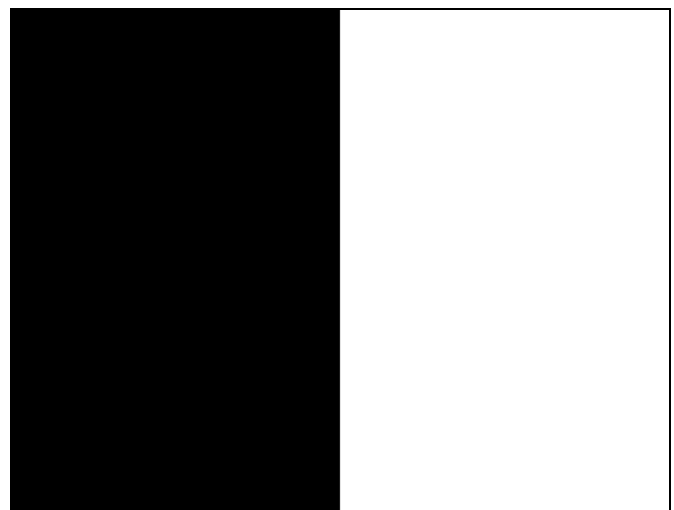

Pour terminer la présentation de ce corps d'hypothèses, nous proposons que la composition de capital social la plus efficiente est celle qui permet aux agents d'être le moins soumis à la tyrannie des liens forts et à l'unicité de la source d'information ${ }^{10}$. Dans notre cas, c'est la combinaison $\mathrm{C}_{4}$ qui présente cette propriété. Enfin, puisque les deux routines sont parfaitement symétriques, nous pouvons définir sur la base du préordre suivant:

$$
\left(\mathrm{C}_{1} \sim \mathrm{C}_{2}\right)<\left(\mathrm{C}_{3} \sim \mathrm{C}_{5}\right)<\mathrm{C}_{4}
$$

la séquence probabiliste d'occurrence d'un projet innovant I :

$$
0 \operatorname{Pr}\left(\mathrm{I} \mathrm{C}_{1}\right)=\operatorname{Pr}\left(\mathrm{I} \mathrm{C}_{2}\right)<\operatorname{Pr}\left(\mathrm{I} \mathrm{C}_{3}\right)=\operatorname{Pr}\left(\mathrm{I} \mathrm{C}_{5}\right)<\operatorname{Pr}\left(\mathrm{I} \mathrm{C}_{4}\right)<1
$$

Par conséquent, dans cette configuration initiale (figure 7), les agents situés au niveau des frontières (sociales), au centre et sur les bords, ont le niveau de capital social le plus performant, ici $\mathrm{C}_{3}$ ou $\mathrm{C}_{5}$. Ce sont donc ces agents, associés ici à des entrepreneurs, qui sont susceptibles d'être à la base des projets les plus en

10. Dans le même ordre d'idées, Cowan, Jonard et Zimmermann (2003) montrent que, dans le cadre d'un modèle multi-agent en interactions, la capacité collective à innover reste maximale lorsque les agents s'engagent dans des partenariats complémentaires, c'est-à-dire aux profils de connaissances bien différenciés. Même s'il faut garder à l'esprit que la dissimilarité et la distance cognitive qui séparent les individus restent toujours facteur d'incompréhension; cette distance peut par conséquent être nuisible à la coopération. 
rupture. À l'inverse, ceux dont le capital social est exclusivement composé de partenaires aux pratiques, idées, etc., semblables, ici $\mathrm{C}_{1}$ ou $\mathrm{C}_{2}$, sont le plus exposés au conformisme. En revanche, $\mathrm{C}_{4}$ n'apparaît pas dans cette configuration initiale.

\subsection{Simulations, résultats et interprétations}

Partant de cette distribution, nous cherchons désormais à comprendre comment évoluent les compositions des voisinages et, par conséquent, l'évolution relative du capital social de chaque agent à mesure que ces derniers interagissent intentionnellement entre eux. Autrement dit, il s'agit ici d'établir les conditions comportementales minimales qui favorisent la modification du capital social vers des niveaux plus performants.

Pour ce faire, nous définissons une probabilité de conversion ou de changement d'opinion dès lors qu'un agent interagit avec l'un de ses quatre voisins :

$$
\begin{gathered}
\operatorname{Pr}\left\lfloor\left(\text { adopter } r_{1}\right)_{i, t+1} \mid\left(\mathrm{r}_{0}\right)_{i, t}\right\rfloor=p, \forall \mathrm{i} \in \mathrm{S} \\
\left.\operatorname{Pr} L\left(\text { adopter } r_{0}\right)_{i, t+1} \mid\left(\mathrm{r}_{1}\right)_{i, t}\right\rfloor=1-p, \forall \mathrm{i} \in \mathrm{S}
\end{gathered}
$$

Chacun des agents formule sa décision selon la même règle de comportement $^{11}$ et la composition des voisinages se modifie à chaque période. Le modèle étant parfaitement symétrique, nous nous limitons aux conditions du passage de la routine $r_{0} \rightarrow r_{l}$ ainsi qu' au comportement d'un nombre restreint d'agents. En effet, les agents que nous considérons comme critiques sont ceux qui sont le plus proches de la frontière sociale et dotés du capital social $\mathrm{C}_{3}$ ou $\mathrm{C}_{5}$ : c'est donc leur attitude à l'égard du lien faible qu'ils entretiennent avec la communauté opposée qui détermine à la fois l'impulsion d'un mécanisme séquentiel de recomposition du capital social et, in fine, la dynamique du changement.

Diverses simulations ${ }^{12}$ permettent de déterminer trois régimes : deux cas polaires et un régime intermédiaire.

Le premier régime est celui que nous définissons par le régime des positions acquises, du conformisme ou encore de l'inertie généralisée (figure 8).

11. On dit que la règle de transition est invariante sur l'ensemble du treillis.

12. Les détails de celles-ci sont disponibles auprès de l'auteur.

Revue internationale P.M.E., vol. 17, nº 2, 2004 
FIGURE 8

Régime des positions acquises et de l’inertie généralisée

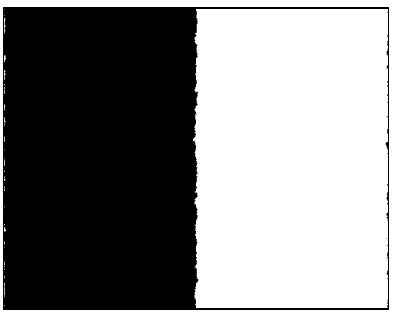

$t=100$

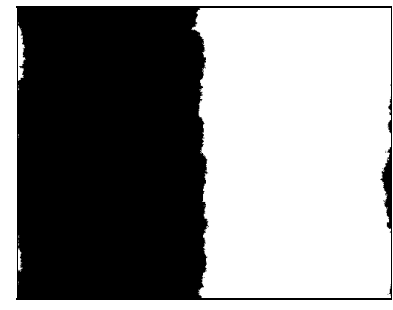

$t=100000$

Il se produit lorsque la probabilité $p$ pour un entrepreneur critique (proche de la frontière) est faible. Autrement dit, la confiance accordée au signal émanant du lien faible est très peu élevée. Les positions sociales ne se modifient guère qu'à la marge et la configuration apparaît méta-stable. Compte tenu que le capital social individuel est relativement peu affecté, seule une minorité des agents sont susceptibles d'innover radicalement à partir d'un stock informationnel performant et discriminant. Enfin, nous pouvons dire que la trajectoire historique individuelle et la position sociale initialement acquises sont ici de forts déterminants du comportement innovant. De ce point de vue, nous formulons la conjecture 1 suivante :

Conjecture 1. La capacité collective à innover radicalement est d'autant plus faible que les liens faibles sont peu activés. L'innovation disruptive est le fait d'une minorité d'entrepreneurs aux positions sociales historiquement acquises ; les comportements conformistes l'emportent.

Le deuxième régime que nous décelons est celui où, au contraire, la ségrégation apparente du premier régime disparaît (figure 9).

Cela se produit lorsque la probabilité $p$ de conversion des agents frontières au signal provenant du lien faible est forte. Autrement dit, la confiance joue ici le rôle de «fluidifiant» et permet, à terme, de réduire les inégalités de composition de capital social. Si ce sont les agents les plus proches de la frontière qui conservent un avantage, en revanche, le degré de porosité de la frontière (un $p$ plus ou moins important) détermine la vitesse à laquelle se diffusent les nouvelles idées. Stratégiquement, les premiers entrepreneurs peuvent avoir intérêt à conserver une rente informationnelle, puisque si ce scénario peut sembler socialement efficient, il est individuellement risqué. Les avantages concurrentiels peuvent être difficiles à maintenir et le risque de voir l'innovation radicale se tarir existe. Nous formulons par conséquent la conjecture 2 . 
FiguRE 9

\section{Régime d'ouverture sociale}

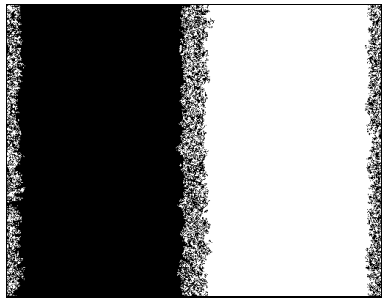

$t=50$

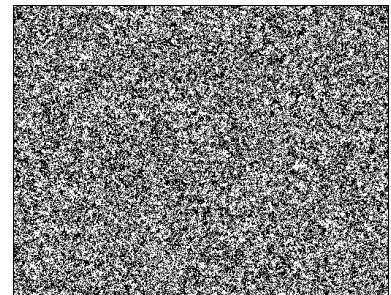

$t>400$

Conjecture 2. Lorsque les liens faibles sont très fortement activés et que la confiance à leur égard est forte, les rentes informationnelles individuelles disparaissent. Socialement efficient, puisque le capital social ne joue plus de rôle discriminant, ce scénario limite très fortement l'occurrence de comportements disruptifs et l'acquisition d'avantages concurrentiels. Une stratégie de rétention ou de diffusion modérée est alors à envisager par les entrepreneurs qui possèdent initialement des liens faibles.

Enfin, le dernier régime, qui est un cas intermédiaire, prévaut lorsque la probabilité $p$ est modérée. Celui-ci laisse apparaître une intéressante propriété d'auto-organisation en cliques sociales (figure 10).

FIGURE 10

Auto-organisation en cliques sociales

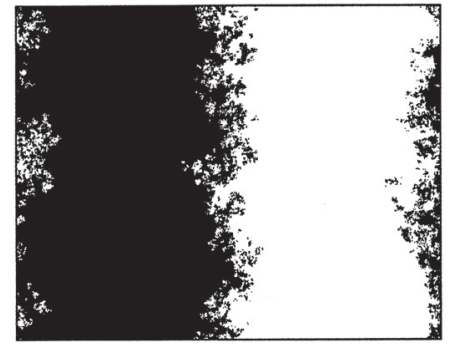

$t=100$

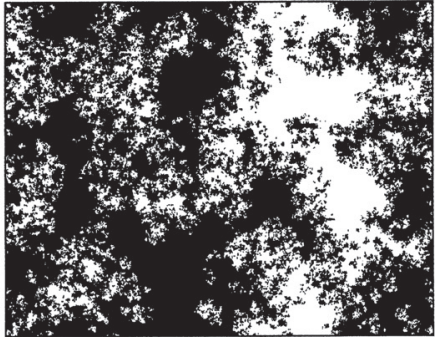

$t=150000$ 
Ainsi, à mesure que la confiance envers les liens forts (dynamique intragroupe) ne prend pas le pas sur les liens faibles (dynamique intergroupes) et que cette confiance accordée à ces derniers n'expose pas l'agent à l'ostracisme, la dynamique sociale de l'innovation est très mouvante. Si la recomposition continue du capital social ne fige pas les positions, elle redistribue aussi les capacités à innover radicalement. S'il demeure une forte cohésion sociale et une forte inertie au sein des cliques plus importantes, en revanche, comme ces dernières se déforment et se recomposent rapidement, les asymétries peuvent ne pas durer un temps infiniment long. Nous formulons ainsi la conjecture 3.

Conjecture 3. Lorsque la confiance accordée aux liens faibles est suffisamment importante mais ne se fait pas au détriment du maintien d'une certaine cohésion, alors les niveaux relatifs de capital social se redistribuent régulièrement limitant dans ce cas les positions acquises. Si la capacité collective à innover est ici maximale, c'est parce que l'innovation disruptive, sans être généralisée, se diffuse de façon à assurer l'acquisition de positions concurrentielles pendant un temps suffisamment long.

Ces diverses simulations exploratoires permettent de poser les fondements théoriques de la mécanique de l'encastrement social de l'innovation, c'est-à-dire l'explicitation de la boucle réseau social $\rightarrow$ confiance $\rightarrow$ capital social $\rightarrow>$ créativité. Sur la base des scénarii qui viennent d'être présentés, nous pouvons désormais mieux cerner ce sur quoi repose la logique de l'encastrement territorial de l'innovation. Autrement dit, nous revenons désormais sur l'articulation des briques élémentaires de la partie gauche de la figure 1.

\section{En guise de conclusion : qu'est-ce que l'encastrement territorial de l'innovation?}

Le recours aux liens faibles, ceux-là mêmes qui supportent la diffusion des opportunités extérieures à la clique d'appartenance, favorise l'adaptabilité individuelle à un environnement évolutif et incertain. La dynamique intercommunautaire et le partage d'expériences sont des forces considérables et décisives. Comme le note fort justement Veltz (2002, p. 88), « faire partie d'un réseau, d'un tissu dynamique, permet d'être dans le coup, de savoir ce qui n'est écrit nulle part, même dans la presse spécialisée, de connaître les réputations des fournisseurs et des clients. Pour un chef d'entreprise, la capacité de jugement, de séparation du positif et du négatif, de pesée qualitative des risques, est fondamentale. Or, ce jugement est infiniment plus difficile pour l'entrepreneur isolé, débutant, socialement enclavé que pour l'entrepreneur bien inséré dans un milieu ». Le capital social, que nous avons défini comme un stock de ressources informationnelles, est un facteur clé de compétitivité 
dès lors qu'il ne se fige pas. Pour ce faire, la confiance ${ }^{13}$ dans les acteurs est un facteur de réduction de l'incertitude qu'un entrepreneur peut être incité à associer à un signal dont l'origine peut être jugée peu crédible. Ainsi, compte tenu que le recours aux liens faibles existe, il devient plus aisé d'absorber les chocs technologiques ou encore commerciaux puisque l'adoption d'une bonne recombinaison de routines est largement facilitée.

La performance, tant individuelle que collective, s'associe donc étroitement à l'activation des liens faibles et, par conséquent, à la confiance qu'on leur accorde. Un territoire qui nierait la performance économique comme le reflet de ce contexte social s'exposerait à s'engager dans des stratégies contre-productives, car c'est bien de l'accessibilité aux réseaux d'échanges et du positionnement en leur sein dont il est question pour chacun, et en être exclu ou ne leur accorder que peu d'importance, c'est très certainement renforcer les inégalités, l'inertie et la marginalisation. Le territoire se doit donc d'être le support des interactions qui favorisent au mieux l'émergence d'un régime social d'innovation efficace.

Il doit pour cela être un fournisseur privilégié de ressources relationnelles et favoriser le passage du premier régime ou du deuxième régime au dernier puisque c'est bien celui-ci qui assure le meilleur compromis entre stabilité des comportements et innovations disruptives individuelles et performance collective. Mettre en mouvement un territoire passe par la rupture avec des pratiques innovantes autorenforçantes qui, le plus souvent, vont mener à l'extrême spécialisation et au risque d'une sensibilité accrue aux chocs (Veltz, 2002). Mais si le capital social est un sucre lent de la compétitivité et détermine fondamentalement l'entrepreneuriat et la créativité, tous les contacts ne se valent pas. Passer son temps à rencontrer son voisin ou les individus d'une communauté de pratiques très proches ne fait que renforcer la probabilité de la survenue d'un régime inertiel et de conformisme. Les comportements en rupture sont, au contraire, le fait d'activations des liens faibles et de rencontres fortuites ou incongrues. La confiance est donc à bâtir ou à renouveler et on ne peut qu'en cela s'associer à Florida $(2001,2002)$ qui plaide pour cette ouverture généralisée des esprits et des lieux de sociabilisation divers et variés.

Les institutions ou le capital institutionnel territorial doivent, d'une part, avoir pour objet de créer les conditions de survenue d'une interaction avec les liens faibles pour le plus grand nombre (des lieux physiques d'échanges) et, d'autre

13. Notons qu'ici nous n'avons pas abordé la question de la construction de la confiance, celle qui en définitive domine la dynamique de la probabilité $p$. L'endogénéisation du processus d'apprentissage de la confiance nécessiterait de s'interroger sur les effets de réputation individuelle, de crédibilité, de prise de risque mais aussi sur un ensemble de comportements cognitifs qui dépassent le cadre de cet article. 
part, de favoriser la confiance à l'égard des sources d'information qui sont, a priori, en rupture forte avec les pratiques quotidiennes. Du point de vue du décideur régional, ce qui compte, ce ne sont pas seulement les localisations d'activités économiques et les différentes externalités technologiques ou pécuniaires qui dynamisent le territoire, mais aussi les externalités relationnelles. Un territoire qui dégage de nombreuses externalités relationnelles contribue très rapidement à élever les coûts de sortie. En effet, en favorisant la réduction simultanée d'une fracture entre les agents fortement imbriqués et ceux plus isolés, il favorise la capacité collective à innover en s'éloignant au maximum des dangers du conformisme. En cela, il présente les caractéristiques d'un territoire attractif et propice à l'entrepreneuriat.

\section{Bibliographie}

Allen, R. (1983), «Collective invention», Journal of Economic and Organization Behaviour, vol. 4, no 1, p. 1-24.

BALA, V. et S. GOYAL (1998), «Learning from neighbours », Review of Economic Studies, vol. $65, \mathrm{n}^{\circ} 3$, p. $595-621$.

BALA, V. et S. Goyal (2000), «A non-cooperative model of network formation », Econometrica, vol. 68, n 5, p. 1181-1229.

BAKER, W. (2000), Achieving Success Through Social Capital, San Francisco, Jossey-Bass.

Cohendet P., P. Llerana, H. Stahn et G. UMBhauer (dir.) (1998), The Economics of Networks, Behaviors and Interactions, Berlin, Springer.

Coleman, J. (1988), « Social capital in the creation of human capital », American Journal of Sociology, no 94, p. S95-S120.

COWAN, R. et N. JONARD (2000), « The dynamics of collective invention », MERIT, cahier de recherche 00-018.

COWAN, R., N. JONARD et J.B. ZIMMERMAnN (2003), «Complémentarités cognitives et production de connaissances nouvelles. Une analyse en termes de réseaux », Revue d'économie industrielle, $\mathrm{n}^{\mathrm{o}} 103$, p. 253-274.

DALlE, J.M. et J. ViCENTE (2001), «L'économie des interactions », Cahier de recherche du LEREPS, Université de Toulouse I.

DAVID, P. (1988), «Putting the past into the future of economics », Technical Report 533, Institute for Mathematical Studies in the Social Sciences, Stanford University.

FLORIDA, R. (2001), «The economic geography of talent », Cahier de recherche, Université Carnegie-Mellon.

FLORIDA, R. (2002), «Bohemia and economic geography », Journal of Economic Geography, vol. $2, \mathrm{n}^{\mathrm{o}} 1$, p. 55-71.

FORAY, D. (2000), L'économie de la connaissance, Paris, La Découverte, coll. « Repères ».

GlAESER, E., D. LAIBSON et B. SACERDOTE (2001), «The economic approach to social capital », Cahier de recherche 1916, Harvard Institute of Economic Research et Cahier de recherche $7728 \mathrm{du}$ NBER. 
GRANOVETTER, M. (2000), Le marché autrement : les réseaux de l'économie, Paris, Desclée de Brouwer.

JACKSON, M. et A. WOLINSKY (1996), «A strategic model of social and economic networks », Journal of Economic Theory, vol. 71, n ${ }^{\circ} 1$, p. 44-74.

Julien, P.-A., E. ANDRIAMBELOSON et C. RAMANGALAHY (2002), «Réseaux, signaux faibles et innovation technologiques dans les PME du secteur des équipements de transport terrestre », Congrès international francophone de recherche en PME, Montréal.

KIRMAN, A. (1992), «Whom or what does the representative agent individual represent?», Journal of Economic Perspectives, vol. 6, n 2, p. 117-136.

KIRMAN, A. et J.B. ZIMMERMANN (dir.). (2001), Economics with Heterogeneous Interacting Agents, Berlin, Springer.

RUEF, M. (2002), «Strong ties, weak ties and islands : structural and cultural predictors of organizational innovation », Industrial and Corporate Change, vol. 11, $\mathrm{n}^{\mathrm{O}} 3$, p. 427-449.

Saxenian, A. (1994), Regional Advantage: Culture and Competition in Silicon Valley and Route 128, Harvard, Harvard University Press.

SCHElling, T. (1978), Micromotives and Macrobehavior, New York, Norton \& Company.

SuIRE, R. (2002), Réseaux sociaux et géographie économique, Thèse de doctorat de l'Université de Rennes I.

VELTZ, P. (2002), Des lieux et des liens : le territoire français à l'heure de la mondialisation, Paris, Édition de l'Aube.

Von HiPPEL, E. (1988), The Sources of Innovation, New York, Oxford University Press.

WATTs, D. (1999), Small Worlds : The Dynamics of Networks Between Order and Randomness, Princeton University Press.

WATTS, D. et S. STROGRATZ (1998), «Collective dynamics of small world networks », Nature, no 393, p. 400-403.

WoOLCOCK, M. (2001), «Le rôle du capital social dans la compréhension des phénomènes économiques et sociaux », ISUMA, vol. 2, nº 1, p. 11-18.

Revue internationale P.M.E., vol. 17, nº 2, 2004

(c) 2004 - Presses de l'Université du Québec

Édifice Le Delta I, 2875, boul. Laurier, bureau 450, Sainte-Foy, Québec G1V 2M2 • Tél. : (418) 657-4399 - www.puq.ca

Tiré de: Revue internationale P.M.E., vol. 17, $\mathrm{n}^{\circ} 2$, sous la direction de Pierre-André Julien • PME1702N

Tous droits de reproduction, de traduction et d'adaptation réservés 